\title{
Childhood predictors of adult mental disorders: implications for prevention
}

\author{
Hemamali Perera
}

\section{Summary}

Longitudinal studies have in the past few years made major contribution to understanding the continuity of childhood mental and behavioural disorders into adulthood. Four themes that predict this childhood to adult continuity are identifiable. Certain temperamental and behavioural traits that are recognizable even at a very young age is known to predict antisocial behaviour later in life. Also, presenting psychopathology in childhood disorders determines the likely psychopathology in adulthood. Common childhood disorders such as attention deficit hyperactivity disorder and autism spectrum disorder though persistent are often misdiagnosed in adults due to the symptom profile being different to that in children. Finally, psychosocial adversities in childhood predict psychopathology in adulthood. Although such knowledge provides valuable insights, efforts on prevention are hampered by poor understanding about mediating factors that determine persistence of disorders. Nevertheless, evidence based early interventions have proven to have some efficacy in reducing childhood impairments that may otherwise persist into adulthood.

SL J Psychiatry 2013; 4 (1): 1-2
The World Health Organization (WHO) identifies three compelling reasons for developing effective interventions for mental and behavioural disorders in children and adolescents. One such reason given is "since there is a high degree of continuity between child and adolescent disorders and those in adulthood, early intervention could prevent or reduce the likelihood of long-term impairment" (1). The recognition of this continuity is relatively recent but knowledge has rapidly expanded. The evidence has come from a range of high quality and large scale longitudinal / epidemiological studies, some using repeat multi-method assessments. Further support is that majority of mental disorders in adults are recognized as developmental in nature. Of those who showed mental health problems at 26 years, $50 \%$ had met criteria for a mental disorder by 15 years of age. By late adolescence, this figure had reached $75 \%(2,3)$.

From the evidence provided by the longitudinal studies, four themes can be identified to understand the predictors of disorders from childhood to adult. First are the temperamental and behavioural traits identifiable in children very early in life. Such vulnerabilities can be recognized as early as 2-3 years. In a follow up of a birth cohort, impulsive, restless and distractible (under-controlled) 3 year olds were more likely to meet diagnostic criteria for antisocial personality disorder and to be involved in crime at 21 years. Similarly, shy, fearful, easily upset (inhibited) 3 year olds were more likely to be diagnosed with depression. Both groups were more likely to attempt suicide and have alcohol-related problems. Controls for family social class did not change the findings (4). These findings were replicated where 3 year olds with soiling, daytime enuresis and externalizing behaviour were associated with adult conviction for violent offenses (5). In schizophrenia, longitudinal studies have found that social, emotional and behaviour problems were more likely to be present in childhood. In addition, delay in motor coordination, impaired receptive language and cognitive functions were detectable as early as second year of life. All these early features were independent of socioeconomic, obstetric and maternal factors (6).

Secondly, the type of presenting psychopathology in childhood determines the likely psychopathology in adulthood. Paediatric obsessive compulsive and phobic anxiety can become chronic conditions that persist. Anxious- disruptive girls and disruptive boys are more likely than their peers to attempt suicide by early adulthood (7). Recurrence rate of major depression in pre-pubertal childhood in a 21 year follow up was $62.6 \%$ (8). Children with comorbid conduct disorder showed an overall higher rate of any affective disorder. However, such psychopathological precursors in childhood may not always directly predict the adult disorder. For example, sometimes childhood anxiety predicts depression in adult rather than anxiety (9).

Thirdly, certain disorders familiar in childhood, but not often considered in the diagnostic profiles of adult psychiatrists, continues to adulthood. Attention deficit hyperactivity disorder (AHD) and autism spectrum disorder (ASD) are two such disorders. Adult ADHD, now a well recognized entity, shows persistence of inattention rather than hyperactivity with disorganisation, forgetfulness and poor time management skills. More relevant to management in adults are the associated psychosocial problems and comorbidities such as increased risk of substance use disorders, relationship and employment problems and law-breaking behaviour (10). In ASD, positive features are less evident in the reciprocal social interactions and restricted and repetitive behaviour domains but communication, socializing and difficulties in making friendships continue (11).

Finally, psychosocial adversities experienced in childhood has been recognized as a significant predictor of psychopathology in adulthood. Whereas childhood socioeconomic hardships predicted onset of anxiety, mood, substance use and behavioural disorders in adult, low parental education were more predictive of their persistence and severity (12). Victims of bullying in childhood are reported to have a higher risk of generalized anxiety, panic disorder and agoraphobia and suicidality in young adulthood after accounting for childhood psychiatric problems and family dysfunction (13). Similarly, bullies persist to antisocial personality disorder. It is also possible that children with preexisting anxiety become victims of bullying more often and those with traits persisted into adulthood irrespective of their experiences. At the same time, there is substantial literature to support the impact of abusive experiences in childhood and their long-term serious negative consequences on mental health and social well being, extending well into adulthood. 
The knowledge available from longitudinal studies leaves child and adolescent mental health services with many challenges regarding prevention. Implementing effective intervention in childhood disorders to reduce incidence of psychiatric disorders in the adult population would be the primary challenge here. At the same time, there are gaps in the knowledge where information with much relevance to prevention is missing. One such knowledge gap is why many with childhood disorders and psychopathology do not become disordered adults. The mediating factors, whether developmental, genetic or environmental or their combination that determine the continuity or discontinuity is not being well understood is a serious barrier to prevention. Genetic liabilities make children more vulnerable to stress, either through their higher sensitivity or by behaving in ways that invite stress into their lives. Such processes can add more psychopathology to the equation and in turn facilitate continuity. At the same time, persistence of psychopathology and disorders into adulthood would mean that nonintervention in childhood results in a more lengthy "duration of untreated illness" and the likelihood of a poorer clinical and functional outcome. On the other hand, ignorance, stigma and misinterpretation of symptoms, poor access to mental health care have all contributed to a large treatment gap in children and adolescents, posing a major barrier to early intervention. There is ample evidence to support that early developmental intervention in functional impairment, cognitive or social or in any other domain, can make a significant difference to later quality of life. Such intervention is especially justified if there is a familial risk as well, such as schizophrenia. Preventive efforts should focus on reducing antenatal and perinatal risks, which needs direct involvement of primary healthcare services. Targeted prevention programmes are necessary in socioeconomically deprived families due to the specific vulnerabilities recognized in these children, where close collaboration between mental health and social welfare systems is essential. In addition to medication for mood dysreguation and poor impulse control, interventions in antisocial and disruptive behaviour in children requires strengthening parenting skills, improving academic outcome, social skills training and preschool or school-based care. Unfortunately, such programmes are not effective in adolescents unless with involvement of the juvenile justice system. Though high resource-utilizing in nature, such multisystemic approaches are favoured for their evidence based outcomes. Nevertheless, the main responsibility falls on the child mental health services to clinically intervene and also educate and train relevant persons, and also ensure smooth transition to adult psychiatry services to prevent breakdown of treatment regimes.

\section{Declaration of interest}

None declared

Hemamali Perera, Professor in Psychiatry

Department of Psychological Medicine, Faculty of

Medicine, University of Colombo, Sri Lanka

E mail: hemamali_p@yahoo.com

\section{References}

1. World Health Organization. Child and Adolescent Mental Health Policies and Plans. Mental Health Policy and Service Guideline Package. WHO Geneva 2005

2. Kim-Cohen J, Caspi A, Moffitt T, Harrington H, Milne B, Poulton R. Prior juvenile diagnoses in adults with mental disorder: Developmental follow-back of a prospectivelongitudinal cohort. World Health Organization. Child and Adolescent Mental Health Policies and Plans. Mental Health Policy and Service Guideline Package. WHO Geneva 2005

3. Kessler RC, Berglund P, Demler O, Jin R, Merikangas $\mathrm{KR}$, Walters EE. Lifetime prevalence and age-of-onset distributions of DSM-IV disorders in the national comorbidity survey replication. Arch Gen Psychiatry 2005;62(6):593-602

4. Caspi A, Moffitt TE, Newman DL, Silva PA. Behavioral observations at age 3 years predict adult psychiatric disorders. Longitudinal evidence from a birth cohort. Arch Gen Psychiatry 1996;53(11):1033-1039

5. Stevenson J, Goodman R. Association between behaviour at age 3 years and adult criminality. B J Psychiatry 2001;179:197-202

6. Isohanni, M., Murray, G., Jokelainen, J., Croudave, T., \& Jones, P. The persistence of developmental markers in childhood and adolescence and risk for schizophrenic psychoses in adult life: A 34 year follow-up of the northern Finland 1966 birth cohort. Schizophr Res 2004;71:213-225

7. Brezo J, Barker ED, Paris J, Hébert M, Vitaro F, Tremblay RE, Turecki G. Childhood trajectories of anxiousness and disruptiveness as predictors of suicide attempts. Arch Pediatr Adolesc Med 2008;162(11):1015-1021

8. Weissman, M.M., Wolk, S., Wickramaratne, P., Goldstein, R.B., Adams, P. Children with prepubertal-onset major depressive disorder and anxiety grown up. Arch Gen Psychiatry 1999;56:794-801

9. Copeland WE, Adair CE, Smetanin P, Stiff D, Briante C, Colman I, Fergusson D, Horwood J, Poulton R, Jane Costello E, Angold A. Diagnostic transitions from childhood to adolescence to early adulthood. J Child Psychol Psychiatry 2013; Mar 2. doi: 10.1111/jcpp.12062. [Epub ahead of print]

10. Wilens TE, Faraone SV, Biederman J: Attention-deficit/ hyperactivity disorder in adults.JAMA 2004; 292(5):619-623

11. Seltzer MM, Krauss MW, Shattuck PT, Orsmond G, Swe A, Lord C. The symptoms of autism spectrum disorders in adolescence and adulthood. J Autism Dev Disord 2003;33(6):565-81

12. McLaughlin KA, Breslau J, Green JG, Lakoma MD, Sampson NA, Zaslavsky AM, et al. Childhood socioeconomic status and the onset, persistence, and severity of DSM-IV mental disorders in a US national sample. Soc Sci Med 2011;73(7):1088-1096

13. Copeland WE, Wolke D, Angold A, Costello EJ. Adult Psychiatric Outcomes of Bullying and Being Bullied by Peers in Childhood and Adolescence. JAMA Psychiatry 2013;70(4):4192003;60:709-717 